\title{
Critical Care for the Patient with Ebola Virus Disease (EBV): The Nebraska Perspective
}

\section{Summary}

Care for patients with Ebola Virus Disease (EVD) and other highly infectious diseases is demanding and requires an unwavering commitment to teamwork and stringent infection control measures. Patients with EVD are severely ill and require specialized critical care interventions. The care of these patients is resource intensive from a clinical, technical, emotional, and financial perspective. Successful treatment of these patients requires facilities to be fully prepared for the intense resource demand associated with their admission and continued cares.

Received: October 05, 2015; Accepted: November 19, 2015; Published: November 26, 2015

\section{Introduction}

Last fall the Nebraska Biocontainment Unit (NBU) team was activated and reported to care for a patient being admitted with confirmed Ebola Virus Disease (EVD) [1, 2]. Upon entering the unit, our stress response was apparent as we noted that our hearts sped up, our palms became diaphoretic, and we had to ask ourselves, "Is this really happening? Ebola in the United States?" Critical care training quickly kicked in, the fears receded and we recognized that we were exactly the right people to respond to this patient's needs. As health care providers we are called to do that which very few other career paths require, which is to put someone else's needs ahead of our own. We strive to learn all we can about the human body so that we may help heal others, to provide not only physical healing but emotional support, and above all else, to do no harm. As healthcare providers who volunteer to work in the NBU, those desires drive us to go one step further, to provide that same level of care to patients for whom no one else can [3].

Recent experiences in treating EVD in the United States provided a unique opportunity to see how the application of a resource rich environment and the ability to provide an intensive level of care can change the outcome of this highly lethal disease. The provision of this care has changed the question from "Should we provide an aggressive critical care approach for the treatment of EVD" to "How will we provide an aggressive critical care approach for the treatment of EVD?" Many healthcare providers are fearful of patients with EVD and this is a major barrier to optimal care. A healthy respect for the highly infectious nature of this disease is valid, although there are strategies and equipment available that can effectively mitigate the potential for inadvertent exposure

\section{Angela Vasa, Michelle Schwedhelm and Daniel Johnson}

\author{
Emergency Preparedness \& Infection \\ Prevention, 987422 Nebraska Medical \\ Center, USA
}

Corresponding author:

Michelle Schwedhelm

\section{- SSchwedh@nebraskamed.com}

Emergency Preparedness \& Infection Prevention, 987422 Nebraska Medical Center, USA

Citation: Rega P, Brian Fink. AED Education: A Dilemma for Public Health and a Challenge for Critical Care Specialists. J Intensive \& Crit Care 2015, 1:1.

during the provision of clinical care [4]. In this article, we hope to communicate effective strategies for preparing healthcare workers for the mental, physical and technical challenges of caring for patients with EVD.

\section{Training}

Preparing for the provision of intensive care unit (ICU) level of care for patients infected with EVD can present many challenges. These patients often require interventions that are considered invasive and involve the skills of trained intensivists, as well as nurses proficient in managing the ongoing care of patients with this high level of acuity [5]. The interventions must be implemented while wearing advanced levels of personal protective equipment (PPE) (Figure 1a and $\mathbf{1 b}$ ), thus potentially limiting the dexterity of the providers involved. This consideration must be accounted for in the training and preparation provided to staff prior to the admission of an actual patient. Providing routine training for key personnel provides the opportunity to gain confidence in their ability to perform the procedures, as well as to build a firm foundation of processes for many aspects of care. The NBU had the benefit of training and conducting drills for multiple years prior to the admission of the first patient. This span of time allowed for the development and refinement of specific policies and procedures, addressing critical issues including, but not limited to, the insertion of central venous catheters, endotracheal 


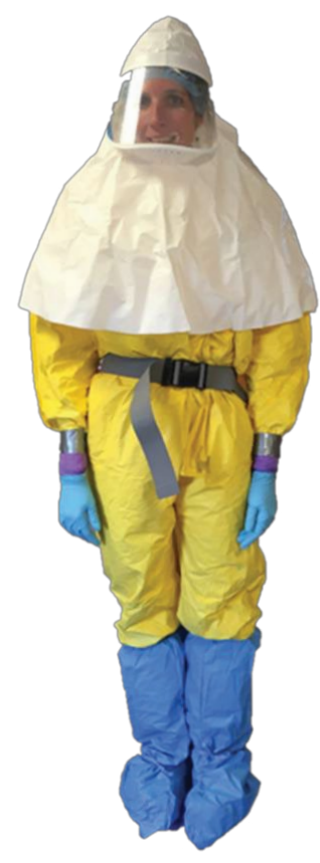

Figure 1a Powered Air Purifying Respirator (PAPR) Level PPE.

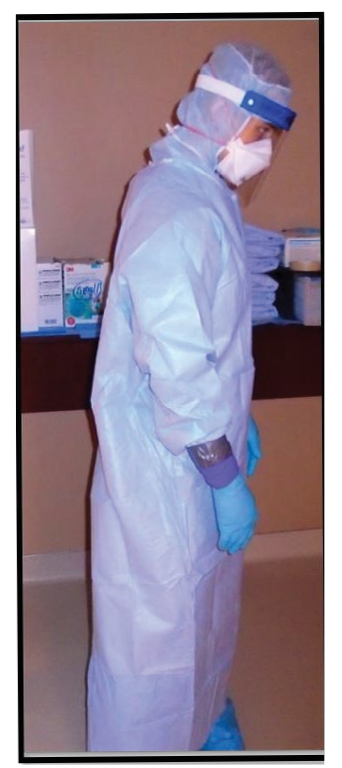

Figure 1b High Level PPE

intubation, the use of continuous renal replacement therapy, execution of advanced cardiovascular life support (ACLS) and the extraction and provision of care for a provider who has a medical emergency in the patient care area. Developing and exercising detailed policies to guide cares within the unit, as well as maintaining an expert staff are key components to operating a high-level biocontainment unit.

\section{Care Delivery}

The development of appropriate staffing models that includes expert consultation with an interprofessional team is essential to successfully treat patients with EVD. The NBU nursing staff is comprised of a wide range of specialties, including critical care, pediatrics, operating room, labor and delivery, interventional radiology, telemetry and the emergency department. We believe that this tremendous diversity in backgrounds and skill sets benefits our patients, as the nurses are able to learn new concepts and skills from colleagues during training exercises and during patient care. In addition to nurses we benefit from the critical care respiratory therapists and patient care technicians who complement the team.

The provision of care for patients with EVD is complex and must account for the high level of staff preparation needed to safely provide the care necessary. It is imperative that a just culture be fostered and maintained. In a just culture the focus is on effective teamwork to accomplish the goal of safe, high-quality patient care [6]. In order to provide care for patients with EVD all staff must feel empowered to identify and take action to prevent errors from occurring, and to improve the patient care environment. Many tools are utilized within the NBU to assist with adherence to the policies and to maintain a safe work environment. The donning and doffing procedures require both vigilance and attention to detail. The importance of following the policy while performing these procedures guided the development of checklists to serve as a guide while performing these functions. (Figures $\mathbf{2} \mathbf{a}$ and 2b) The presence of these lists serves as a reference tool and provides a means of reinforcement when providing guidance to a team member. All staff members, regardless of title or position, are expected to hold one another accountable for adhering to the policies and procedures within the NBU.

Critical care physicians work collaboratively with infectious disease physicians in order to provide comprehensive care for patients with EVD $[7,8]$ Additionally, consulting services such as nephrology are utilized as necessary for the implementation and management of continuous renal replacement therapy. Patients with EVD receive the same high level of care that all ICU patients receive, with attention paid to evidence-based best practices, objective risk-versus-benefit assessment clinical care prior to implementing proposed interventions and the utilization of subspecialty consultation as needed.

Each patient admitted to the NBU at Nebraska Medicine presented at a different stage of the disease process. All three confirmed EVD patients required aggressive fluid resuscitation with frequent lab monitoring to adequately equilibrate electrolyte disturbances. One patient that was admitted later in the disease process (day 14 of illness) suffered from multi-organ system failure as a result of combined septic shock and hypovolemic shock. Preliminary data from resource-rich healthcare environments suggest that early provision of aggressive supportive care increases the likelihood of survival for patients with EVD.

\section{Establishing Central Venous Access}

Central venous catheter placement is indicated because of the need for large volume resuscitation, electrolyte repletion, experimental therapeutic interventions, frequent laboratory sampling and continuous monitoring of central venous pressure. 
Figure 2a Donning High Level PPE

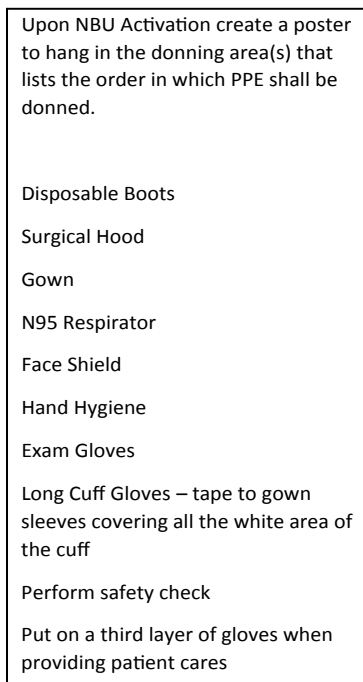

Figure 2a

NBU Ordered Guide for Donning High Level PPE

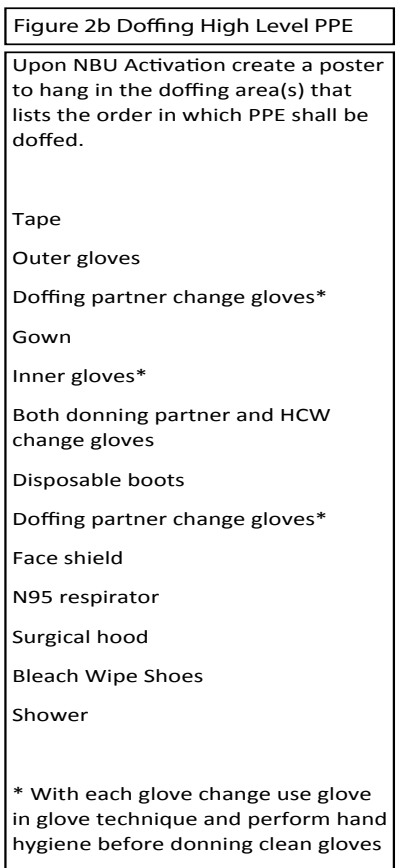

Figure 2b NBU Ordered Guide for Doffing High Level PPE

Our center established central venous access on day 1 of care. Early placement of a central venous catheter greatly reduced the additional risk of exposure for staff throughout the clinical course by decreasing the need for frequent venipuncture for phlebotomy and peripheral IV placement. It also decreased the risk of bleeding from multiple phlebotomy sites. When providing care for a patient requiring multiple intravenous solutions at the same time the presence of a central venous catheter simplifies the delivery of this care. This invasive procedure required providers to have proficient technical skills for line placement. In the NBU this procedure merits the use of enhanced PPE, including a coverall due to the increased splash risk inherent in placing central venous access. Strict adherence to sterile technique must be observed. This warrants the application of sterile attire in addition to the biological PPE worn.

\section{Continuous Renal Replacement Therapy}

Prior to the first patient being admitted, the NBU leadership team discussed at length the potential use of continuous renal replacement therapy (CRRT), as patients with Ebola have been known to progress to anuric acute renal failure. Risk-benefit analyses were conducted with regard to CRRT and other advanced ICU modalities such as endotracheal intubation and mechanical ventilation. The use of CRRT requires that skilled practitioners be proficient in the operation of the continuous dialysis machine. Early inclusion of critical care physicians, nephrologists and critical care nurses on our team allowed us to seamlessly integrate these interventions into our plan of care. Special considerations in the use of CRRT for an EVD infected patient in our unit included the decision to pre-treat the effluent before releasing it into the waste stream, modifying our staffing ratios once treatment was initiated, providing refresher training to our staff related to the CRRT system and including our lab personnel in discussions regarding the high frequency of necessary laboratory testing. Nephrologists were contacted well in advance to secure an agreement for consultation in order to provide expert input in the event that CRRT was needed. Our ability to work as a team to mitigate the challenges related to the implementation of CRRT allowed us to smoothly provide this advanced intervention when it was clinically indicated.

\section{Advanced Airway Intervention}

Endotracheal intubation and the use of mechanical ventilation carry additional challenges for patients with EVD. The increased risk of exposure when performing a potentially aerosol generating procedure warranted an in-depth discussion related to the process that would be used if intubation was indicated. NBU leadership sought the airway expertise of the Department of Anesthesiology in the creation of optimal airway management protocols. The presence of experienced critical care anesthesiologists and respiratory therapists on our team allowed for a comprehensive analysis of this intervention prior to policies being written. Special consideration was given to the types of medications that would be used, the number of staff that would be needed in the room and the type of PPE those providers would utilize. Intubation, a potentially aerosol generating procedure, necessitated the use of a higher level of PPE. This included a coverall suit with a PAPR for any healthcare provider who might be near the patient's airway during intubation. Due to the risk of aerosolization during intubation in the event of coughing or vomiting, our critical care anesthesiologists recommended rapid sequence induction with use of a rapid-onset hypnotic agent followed immediately by a rapid-onset neuromuscular blocking agent. This is the method utilized for intubation in the NBU.

Anesthesiologists at Nebraska Medicine strongly advocated for avoidance of emergency or "stat" intubations on patients with EVD, due to the need for advanced preparation of equipment and medications, systematic donning of PPE and the need for 
a controlled environment to minimize risk to the patient and to healthcare workers. NBU policy states that the attending anesthesiologist on call will respond for all intubations for patients with EVD. This policy required the hospital to provide advance training in donning and doffing of appropriate PPE for all anesthesiologists. In the creation of intubation policies, NBU leadership balanced the desire to minimize the number of staff in the patient's room with the need to provide adequate nursing and respiratory therapy support for the anesthesiologist performing the intubation. NBU leadership also recognized that following the transition to positive pressure ventilation, increased staffing would be necessary. With these considerations in mind, the NBU team made the decision to staff two critical care nurses as well as one critical care respiratory therapist in the room during intubation and for the immediate period following.

\section{Staffing the Nebraska Biocontainment Unit}

NBU leadership evaluated staff competency levels, experience and critical thinking skills and used this information as the foundation for creating a safe patient care environment. When determining staffing needs for the unit, attention was given to patient acuity, the specialized skill set needed for that acuity, anticipated clinical interventions, and staff availability. When developing the staffing schedule for the NBU, the number of nurses, therapists, care techs and physicians scheduled for each shift was dependent upon the number of roles needed to be filled. The NBU used a twelve hour shift model with the assumption that each nurse would provide in-room care for the patient in four hour increments. The decision to establish a four hour increment of care while wearing advanced PPE was based upon the experiences of our staff during numerous drills. Direct feedback from staff regarding their comfort and tolerance of the PPE drove this decision. This necessitated the inclusion of no less than three nurses on duty for each shift. In addition, a minimum of three staff members were added for the day shift, and two for the night shift, which could be any combination of nurses, respiratory therapists or patient care technicians. The discrepancy in staffing ratios from day to night shift was driven by our use of the autoclave, which is used to decontaminate all waste generated within the NBU. The autoclave was operated solely during the day shift, which calls for a dedicated staff member in full PPE. The additional staff members on each shift were used to fill defined roles, including a dedicated doffing partner, a donning partner and a "tasker". The roles outside of the in-room nurse were assumed on a rotating basis by all staff as every member of our team is thoroughly trained in each role.

When determining staffing levels for a specialized area, such as the NBU, standard staffing matrices are not applicable and leadership must consider current evidence and staff input to develop a successful staffing model. Core NBU staff members and NBU leadership worked together to establish a staffing model that suited the specific requirements for providing care to patients with EVD. The diverse nature of our team allowed us to tailor our staff scheduling to most closely match the needs of the patient in order to provide optimal care.

\section{Conclusion}

Advanced planning for critical care interventions in the NBU allowed us to seamlessly implement all necessary measures when providing care for patients with EVD. While the use of these interventions does not guarantee the successful treatment of $E V D$, withholding these interventions almost certainly reduces the likelihood of survival. The goal of the NBU is to provide advanced medical care and treatment to all patients with highly infectious diseases, consistent with our hospital's standard of care. As critical care health care providers in the NBU, we continue to advance our education and train rigorously to improve our own skills and capabilities, and to elevate the knowledge and expertise of colleagues around the world who might be called upon to care for patients with highly infectious diseases. 


\section{References}

1 Beam EL, Boulter KC, Freihaut F, Schwedhelm S, Smith PW (2010) The Nebraska experience in biocontainment patient care. Public Health Nurs 27: 140-147.

2 Centers for Disease Control and Prevention (CDC) (2015) Ebola virus disease information for clinicians in U.S. healthcare settings.

3 Schwedhelm S, Beam EL, Morris RD, Sebastian JG (2015) Reflections on interprofessional team-based clinical care in the ebola epidemic: the Nebraska Medicine experience. Nurs Outlook 63: 27-29.

4 Wallace BC (2013) Nurse staffing and patient safety: what's your perspective? Nurs Manage 44: 49-51.
5 Barnsteiner J (2011) Teaching the culture of safety. The Online Journal of Issues in Nursing 16: 5.

6 Clark S, Donaldson N (2008) Nurse staffing and patient care quality and safety: An evidence based handbook for nurses. (Chapter 25). Rockville, MD: Agency for Healthcare Research and Quality, USA.

7 Johnson DW, Sullivan JN, Piquette CA, Hewlett AL, Bailey KL, et al. (2015) Lessons learned: critical care management of patients with Ebola in the United States. Crit Care Med 43: 1157-1164.

8 Kortepeter MG, Smith PW, Hewlett A, Cieslak TJ (2015) Caring for patients with Ebola: a challenge in any care facility. Ann Intern Med 162: 68-69. 